\title{
The implications of pharmacogenomics in the treatment of HIV-I-infected patients of African descent
}

This article was published in the following Dove Press journal:

Pharmacogenomics and Personalized Medicine

9 September 2009

Number of times this article has been viewed

\section{Hector Clarke \\ Shaker A Mousa}

The Pharmaceutical Research Institute, Albany College of Pharmacy and Health Sciences, Rensselaer, NY, USA
Correspondence: Shaker A Mousa The Pharmaceutical Research Institute at Albany, Albany College of Pharmacy and Health Sciences, I Discovery Drive, Room 238, Rensselaer, NY I 2 I44, USA

$\mathrm{Tel}+\mathrm{I} 5186947397$

Fax + I 5186947567

Email shaker.mousa@acphs.edu
Abstract: One of the great advances in the treatment of HIV-1 infection was the development of the highly active antiretroviral therapy (HAART). Although this treatment strategy is highly effective in many individuals, interpatient variability of drug response and high incidences of short- and long-term toxicities remain significant problems associated with this treatment. Logically, pharmacogenetic differences among HIV-1-infected individuals are thought to represent important factors contributing to antiretroviral drug response. Studies have identified polymorphisms in drug-metabolizing enzymes, drug transporters, and most recently the human leukocyte antigen locus that appears to have significant effects on the clinical outcomes of antiretroviral therapy. Furthermore, some studies have shown that many of these crucial polymorphisms are more likely or less likely in certain populations. This review investigates the potential role of pharmacogenomics in the management of HIV-1 infection in people of African descent.

Keywords: HIV, AIDS, pharmacotherapy, pharmcogenomic, genetics, African populations, personalized medicine

\section{Introduction}

HIV/AIDS has claimed the lives of more than 25 million people worldwide, mostly within Africa. There have been remarkable advances in the treatment of HIV/AIDS during the last 20 years. Today there are over 20 antiretroviral agents representing five therapeutic drug classes, each with a unique mechanism of action. ${ }^{1}$ The first class of drugs that were used in antiretroviral therapy was nucleoside/nucleotide reverse transcriptase inhibitors (NRTIs). This class of drugs inhibits reverse transcription by becoming incorporated into viral DNA while it is being synthesized thereby preventing elongation. Popular drugs in this class include zidovudine, lamivudine, and abacavir. Nonnucleoside reverse transcriptase inhibitors (NNRTIs) are another class of drugs that also inhibit reverse transcription. NNRTIs, however, achieve this by directly binding to viral reverse transcriptase, inhibiting its function. There are only two widely used drugs in this class, efavirenz (EFV) and nevirapine (NVP). The third major class of antiretroviral drugs are the protease inhibitors (PIs). These drugs inhibit the proteases that are needed by the virus to assemble new virons. Popular PIs include indinavir, ritonavir, and atazanavir. The remaining classes of drugs are integrase inhibitors, which prevent viral DNA from integrating into host DNA, and entry inhibitors, which prevent the virus from binding and fusing with the host cell membrane. The drugs in these classes are not as widely used as NRTIs, NNRTIs, and PIs. They are often used as additions to standard drug combinations or as salvage therapies after other drug classes have been exhausted. ${ }^{2,3}$ 
Highly active antiretroviral therapy (HAART) is the current standard of care for the treatment of HIV-1 infections. Current guidelines recommend that individuals begin on a HAART regime when their CD4 cell count dips into the range of 350-500 CD4 T cell $/ \mathrm{mm}^{2}$. A typical HAART regime for initial therapy will consist of a combination of three or four drugs, usually two NRTIs with either an NNRTI or a ritonavir-boosted PI. Therapy is consider a success if it is able to suppress the viral load to a point below detection. ${ }^{4}$ Together these agents have been used in combination to treat HIV/ AIDS in millions of patients living with the virus. For many patients, this disease, once thought of as a death sentence, can now be thought of as a chronic disease, requiring life-long therapy. ${ }^{5}$ However, despite these successes, there are still many issues that need to be resolved, including the development of drug resistance and toxicity.

Individualization of medicine through the use of pharmacogenomics may help clinicians prescribe the most efficacious drug combination and dose for each of their patients. ${ }^{1}$ This type of optimization will result in increased efficacy and reduced risk for toxicity. Although pharmacogenomics has already made a clinical impact on HIV-1 therapy, the relationship between interindividual variability in response to anti-HIV-1 drugs and racial identity requires further exploration. Race/ethnicity can be a predictor of how an individual may respond to a particular drug due to the fact that alleles for certain genes that affect drug response can be more or less prevalent from population to population. ${ }^{2}$ The purpose of this review is to highlight potential areas where pharmacogenomics may be of use in both maximizing the efficacy and minimizing the toxicity of anti-HIV-1 therapy for individuals of African descent. Paying special attention to this population is of particular importance because individuals of African descent represent a disproportionate number of the new cases of HIV/AIDS that are presently being diagnosed. Additionally, Sub-Saharan Africa still represents the largest population of individuals with HIV/AIDS, and the majority of new cases reported worldwide are in this area. ${ }^{6}$ Unfortunately, however, the majority of clinical research is performed in countries like the United States where individuals of African descent are minorities, and this population is generally underrepresented in research. ${ }^{7}$

Polymorphisms are naturally occurring variants in the structures of genes and the products that they encode. ${ }^{8}$ Polymorphisms in a gene often have no visible or quantifiable affect on the functioning of the protein product. Sometimes, however, variation in a particular gene can result in altered function of the protein product. Polymorphisms can be of importance to pharmacotherapy when they occur in genes encoding drug-metabolizing enzymes, drug receptor proteins, and other proteins involved in drug response or disease progression. A single-nucleotide polymorphism (SNP) is a polymorphism is a single nucleotide difference that exists between two alleles of the same gene. While this change may seem minute, if it is in a critical spot it can vastly alter the functioning of the protein product. Many of the polymorphisms discussed in this article are SNPs. The study of the impact of human genetic variation upon individual responses to antiretroviral drugs is complex. It is difficult to explain the functional impact of human genetic variation in most cases, and more importantly, these polymorphisms do not act independently to influence disease evolution or treatment response. ${ }^{9}$

\section{Pharmacogenomics and hypersensitivity}

Hypersensitivity reactions are a major concern for patients being treated with antiretrovirals. A drug hypersensitivity reaction is an immunological reaction that an individual can develop in response to the administration of a certain drug. There are a wide range of symptoms that can accompany a hypersensitivity reaction, including serum sickness, hypotension, anaphylaxis, nausea, vomiting, diarrhea, and rash. Although only a small percentage of patients will experience hypersensitivity reactions during their treatment, some reactions can be very severe and even fatal. ${ }^{10}$

A number of human leukocyte antigen (HLA) genes have been linked to hypersensitivity reactions to antiretrovirals. HLA genes are found in region of the mammalian genome known as the major histocompatability complex (MHC). ${ }^{11}$ HLA genes are expressed on the cell surface and display self and nonself antigens to T cells. When a drug is administered to a patient there is a possibility that the drug or some metabolite that is generated from the drug will end up being presented on the cell surface by an HLA protein. If the immune system recognizes this antigen as nonself, a hypersensitivity reaction may occur. The MHC itself is divided into three regions: I, II, and III. The HLA-A, HLA-B, and HLA-C genes can be found in region I, whereas HLA-D is located in region II. Genetic variation in HLA-B has been linked to the risk of developing antiretroviral hypersensitivity reactions, especially to the NRTI abacavir. One polymorphism in particular, HLA-B*5701, has been indicated as conferring a high risk for abacavir-induced hypersensitivity reactions. ${ }^{12-15}$ 
Approximately $5 \%-8 \%$ of patients who are treated with abacavir develop a drug hypersensitivity reaction within six weeks of treatment initiation. The symptoms of an abacavir hypersensitivity reaction can include fever, rash, diarrhea, and flu-like aches. In rare cases, abacavir hypersensitivity reactions can be fatal. ${ }^{16}$ Screening for the HLA-B*5701 allele has proven to be a valuable and cost-effective measure that clinicians can take to reduce the incidence of hypersensitivity reactions in their patients. ${ }^{13,15}$

Many of the studies performed in recent years that have reported on HLA-B*5701 offer conflicting data regarding how sensitive this marker is for predicting the occurrence of abacavir-induced hypersensitivity reactions. In a retrospective case-controlled study $(\mathrm{n}=200)$ Hetherington and colleagues found HLA-B*5701 to be present in $46 \%$ of patients with hypersensitivity and in $4 \%$ of controls. ${ }^{14}$ Similarly, in an observational study $(n=63)$, Hughes and colleagues reported that $46 \%$ of abacavir-hypersensitive patients were positive for HLA-B*5701, compared to $10 \%$ of controls. ${ }^{15}$ One study of the Western Australia HIV Cohort $(n=260)$ reported no detected cases of abacavir hypersensitivity in HLA-B*5701-negative patients. ${ }^{17}$ Mallal and colleagues performed a study also utilizing participants from the Western Australia HIV Cohort $(\mathrm{n}=200)$, and found that $78 \%$ of the patients with abacavir hypersensitivity were positive for HLA-B*5701, while only $2 \%$ of the nonhypersensitive patients were positive for HLA-B*5701. ${ }^{18}$

Although HLA-B*5701 screening is undoubtedly valuable in the clinical setting, the discrepancies in the sensitivity of this marker in predicting abacavir hypersensitivity across studies is puzzling. Furthermore, some researchers have suggested that this marker is not useful for predicting abacavir hypersensitivity reactions in certain populations, including individuals of African descent. ${ }^{15}$ Although many studies have included patients of African descent the conflicting results become even more untrustworthy due to small sample sizes. This is a result of the fact that patients of African descent are less likely to develop hypersensitivity reactions to abacavir and are low carriers of HLA *5701 with a prevalence of $2.5 \%$ in the population. ${ }^{19}$ Saag and colleagues effectively addressed these concerns in a study that included 130 Caucasian patients and 69 patients of African descent who had all been classified as receiving a diagnosis of abacavir hypersensitivity. Forty-two Caucasian patients and five patients of African descent in this study and had shown a positive skin patch result, immunologically confirming abacavir hypersensitivity. Control subjects were racially matched and were selected on the basis of showing tolerance to abacavir for 12 weeks or more. ${ }^{12}$
All subjects were screened for HLA-B*5701. Of the patients who had immunologically confirmed abacavir hypersensitivity (via skin patch test) $100 \%$, in both groups (42 Caucasian patients and 5 patients of African descent), were positive for HLA-B*5701. The screen for the whole group revealed that $44 \%$ of the Caucasian patients and $14 \%$ of the patient of African descent who were given the diagnosis of abacavir hypersensitivity were positive for HLA-B $* 5701 .{ }^{12}$

This study suggests that the diagnosis of abacavir hypersensitivity can easily be muddled by other reactions that patients may be experiencing due to additional drugs that they are being administered. Indeed, when the abacavir hypersensitivity was immunologically confirmed, the sensitivity of HLA-B*5701 as a predictor for abacavir hypersensitivity was $100 \%$. Although the number of African American patients included in this study was small with only five in the critical immunologically confirmed group, it still suggests that screening for HLA-B*5701 in this population is valid and crucial.

\section{Pharmacogenomics and pharmacokinetics}

The pharmacokinetics of a particular drug has much bearing on its efficacy and the risk of adverse side effects. Pharmacokinetics refers to the absorption, distribution, rate of action, metabolism, and excretion of routes of an administered drug. A number of genes affecting the pharmacokinetics of antiretroviral drugs will be discussed in this article, including: multidrug-resistant gene-1 (MDR1) and cytochrome P450 (CYP) genes (CYP2B6, CYP3 A4, and CYP3 A5).

MDR1 is a well characterized ABC-transporter of the MDR/TAP subfamily. There are a number of other names used to refer to this protein, including: p-glycoprotein (P-gp), $\mathrm{ABCB}$, and CD243. MDR1 functions as a broad spectrum efflux pump. MDR1 is expressed in most cell types and can be found at many of the important physiological barriers that drugs must traverse (ie, the intestinal epithelium, the capillary endothelium, and the proximal renal tubular cells. Thus, allelic variation at the $m d r l$ locus can have an influence on the absorption, distribution, and elimination of drugs. ${ }^{20}$

Cytochrome $\mathrm{P} 450$ takes part in phase I metabolism. Phase I metabolism can serve to either activate or inactivate a drug, while phase II metabolism usually involves conjugation reactions that serve to deactivate active metabolites/drugs. Phase I metabolism usually involves making the administered drug more polar through hydrolysis, oxidation, or reduction. Most oxidative reactions are carried out by cytochrome P450. 
There 57 functional CYP genes found in the human genome which have been classified into 18 families and 43 subfamilies. The CYP2 and CYP3 families are responsible for the metabolism of drugs and steroids. ${ }^{21}$ This paper will discuss CYP2B6, CYP3 A4, and CYP3 A5 in particular, with respect to the metabolism of antiretrovirals.

Polymorphisms in CYP2B6, CYP3 A4, CYP3 A5, and MDR1 are distributed differentially across Caucasian populations and populations of African descent. The CYP2B6 $516 \mathrm{G} \rightarrow$ T polymorphism can be found in $3 \%$ of Caucasians and $20 \%$ of individuals of African descent. ${ }^{3}$ The CYP3 A $4 * 1 \mathrm{~B}$ and CYP3 A $5 * 3$ polymorphisms are present at a rate of $5.9 \%$ and $91.1 \%$ in Caucasians, respectively and $59.2 \%$ and $28.1 \%$ in individuals of African descent, respectively. ${ }^{22}$ Additionally, the $3435 \mathrm{C} \rightarrow \mathrm{T}$ polymorphisms found in MDR 1 can be found in $49.7 \%$ of Caucasians and $12.6 \%$ of individuals of African descent. ${ }^{22}$ The presence of these polymorphisms has been found to affect the pharmacokinetics of antiretrovirals and is especially important for determining a patient's response to EFV and NVP. Although these polymorphisms have not been found to affect the efficacy of either EFV or NVP, they do have a significant affect on the plasma concentration and half-life of these drugs. The heightened levels and elongated exposure of these drugs increases the amount of adverse side-effects, toxicity, and the need for discontinuation of treatment in patients.

Both Haas and colleagues ${ }^{23}$ and Ritchie and colleagues ${ }^{24}$ found that the $3435 \mathrm{C} \rightarrow \mathrm{T}$ polymorphism in the MDR 1 gene was associated with decreased risk of hepatotoxicity during treatment with NVP. ${ }^{23,24}$ Thus, individuals of African descent are at higher risk for developing hepatotoxicty during NVP treatment due to the low prevalence $(12.6 \%$ vs $49.7 \%$ in Caucasians) of this polymorphism. In two consecutive studies, Haas and colleagues found that homozygousity for thiamine at position 516 in the CYP2B6 gene was associated with greater plasma exposure to EFV during treatment. ${ }^{3,25}$ This result was independently confirmed by Saitoh and colleagues in a study of 71 children undergoing EFV treatment. ${ }^{26}$ Ribaudo and colleagues measured EVF concentrations after treatment discontinuation. These researchers found that homozygousity for thiamine at position 516 in the CYP2B6 gene resulted in detectable EFV concentrations in $29 \%$ of patients, which was a significantly high percentage than individuals who were heterozygous or homozygous for guanine at this position (5\%). ${ }^{27}$ Greater plasma exposure increases the risk of individuals developing central nervous system side-effects to EFV. Individuals of African descent are therefore more prone to developing these adverse side-effects because of the increased prevalence of this polymorphism in the population (20\% vs $3 \%$ in Caucasians).

\section{Pharmacogenomics and atherogenic dyslipidemia}

Many patients undergoing antiretroviral therapy develop atherogenic dyslipidemia which can put them at risk for experiencing acute cardiovascular events such as myocardial infarction, stoke, deep vein thrombosis, or pulmonary embolism. ${ }^{28-30}$ The development of atherogenic dyslipidemia is most commonly seen in patients receiving protease inhibitors as part of their antiretroviral therapy. ${ }^{28,31}$ Lipid profiles of affected patients usually include increased low- and very-low-density lipoprotein particles (LDL and VLDL), decreased high-density lipoprotein (HDL) levels, and elevated triglyceride levels. ${ }^{32-34}$ They are most commonly found in patients receiving PI-based HAART regimes, especially those containing ritonavir. ${ }^{31,35}$ It is believed that PIs cause these lipid imbalances by modulating the normal production and degradation of apolipoproteins. ${ }^{32,36,37}$

Lipoprotein particles transport dietary lipids throughout the bloodstream. Lipoprotein particles can be distinguished by the type of apolipoproteins they contain. Apolipoproteins dictate the type of lipids that the particle will carry and also act as receptor ligands and enzyme co-factors, thereby controlling the metabolism and uptake of these particular lipids in various tissues. ${ }^{38}$ Apolipoproteins are divided into a number of major classes (apoA, apoB, apoC, etc.) which may be further divided into several subclasses.

LDL particles each contain a single apoB molecule. High LDL levels contribute to atherogenesis, because LDL particles are the major vehicle which delivers cholesterol from the liver to the site of growing atheromas. ${ }^{39}$ HDL particles, on the other hand, are thought to perform the opposite function, transporting cholesterol away from atheromas in the arteries and back to the liver where it can be excreted or metabolized. The most abundant apolipoproteins found in HDL are apoA-I and apoA-II. ApoA-IV is also found in HDL particles and, along with apoA-I, is an important player in terms of cholesterol and triglyceride levels. ${ }^{40}$ Apolipoprotein $\mathrm{C}$ (apoC) is found in VLDLs. ApoC-III is a member of the apoC family which can be found in HDL, LDL, and VLDL particles. ApoC-III inhibits lipoprotein lipase, thereby delaying the breakdown, metabolism, and hepatic clearance of triglyceride-rich lipoprotein particles. ${ }^{40}$ Logically, increases in the levels of apoC-III result in elevated triglyceride levels. SNPs in the promoter region of apoC-III at positions -455 $(\mathrm{C} \rightarrow \mathrm{T})$ and $-482(\mathrm{~T} \rightarrow \mathrm{C})$, as well as a SNP at position 
$3238(\mathrm{C} \rightarrow \mathrm{G})$ of the 3' untranslated region (the Sst I allele), have been associated with hypertriglyceridemia. ${ }^{41,42}$ ApoA-I, apoC-III, and apoA-IV are in linkage disequilibrium with each other, as they are found in a cluster on chromosome $11.43,44$ Apolipoprotein E (apoE) is found in chylomicrons, VLDL, and HDL particles and is involved in the transport and clearance of remnant lipoprotein particles. Associations between SNPs in apoE (the apoE $\varepsilon 4$ and apoE $\varepsilon 2$ alleles) and hyperlipidemia/cardiovascular events have been reported in non-HIV-infected individuals. ${ }^{45}$

Tarr and colleagues performed a study $(n=329)$ that analyzed three SNPs found in the ApoC3 gene $(-455 \mathrm{~T} \rightarrow \mathrm{C},-482 \mathrm{C} \rightarrow \mathrm{T}$, and Sst [3238] $\mathrm{C} \rightarrow \mathrm{G})$ as well as the apoE $\varepsilon 4$ and apoE $\varepsilon 2$ alleles and concluded that individuals with unfavorable genotypes of ApoC3 or ApoE were at the highest risk for developing extremely high triglyceride levels while receiving antiretroviral therapy containing protease inhibitors. ${ }^{46}$ Fauvel et al similarly found that the three polymorphisms of ApoC3 were positively associated with developing atherogenic dyslipidemia during antiretroviral therapy with protease inhibitors $(n=60) .{ }^{47}$ An ambitious study by Foulkes and colleagues $(n=626)$ analyzed five different polymorphisms found in ApoC3: $455 \mathrm{~T} \rightarrow \mathrm{C},-482 \mathrm{C} \rightarrow \mathrm{T}$, Sst [3238] $\mathrm{C} \rightarrow \mathrm{G}$, intron I $\mathrm{G} \rightarrow \mathrm{C}$, and Gly34Gly $\mathrm{C} \rightarrow \mathrm{T}$. The goal of this study was to determine if there was evidence for race/ethnicity-specific effects on plasma lipid levels during HAART treatment. It has already been established that in the general population African Americans, on average, have more favorable lipid profiles than their Caucasian counterparts. ${ }^{48}$ Foulkes and colleagues found variation in the distribution of ApoC3 polymorphisms among Caucasians and individuals of African descent. ${ }^{49}$ The frequencies of these polymorphisms in the two different racial/ethnic is summarized in Table 1. This study confirmed race as a significant predictor of baseline plasma lipid levels; individuals of African descent had lower levels of triglycerides and non-HDL cholesterol. Additionally, this study demonstrated that variations in the ApoC3 gene, distributed differentially among Caucasians and individuals of African descent, influenced the development of dyslipidemia during antiretroviral therapy with protease inhibitors. Although the patients of African descent started with the lowest baseline triglyceride levels, this group showed the greatest increase in triglyceride levels during protease inhibitor exposure. ${ }^{49}$ Although this study does not provide any clear directives for clinical practice, it has provided evidence to justify similar studies. The data presented clearly indicates that conclusions from pharmacogenomic studies cannot be generalized across populations, and that the variable of race must be investigated before guidelines are written.

Table I Frequency of polymorphisms that affect antiretroviral drug response in Caucasians and individuals of African descent.

\begin{tabular}{|c|c|c|c|}
\hline \multicolumn{4}{|c|}{ HLA-dependent hypersensitivity reactions } \\
\hline \multirow[t]{2}{*}{ Drug(s) } & \multirow[t]{2}{*}{ Polymorphism } & \multicolumn{2}{|l|}{ Prevalence } \\
\hline & & Caucasian & African descent \\
\hline Abacavir & HLA-B*570I & $5.3 \%^{12}$ & $2.5 \%^{12}$ \\
\hline \multicolumn{4}{|c|}{$\begin{array}{l}\text { Adverse reactions resulting } \\
\text { from altered pharmacokenetics }\end{array}$} \\
\hline EFV, NVP & $\mathrm{CYP2B} 6516 \mathrm{G} \rightarrow \mathrm{T}$ & $3 \%^{3}$ & $20 \%^{3}$ \\
\hline EFV, NVP & MDRI $3435 C \rightarrow T$ & $49.7 \% \%^{22}$ & $12.6 \% 22$ \\
\hline EFV, NVP & CYP3 A4*IB & $5.9 \%^{22}$ & $59.2 \% 22$ \\
\hline EFV, NVP & CYP3 A5*3 & $91.1 \% 22$ & $28.1 \% 22$ \\
\hline \multicolumn{4}{|c|}{ Atherogenic dyslipidemia } \\
\hline Protease Inhibitor & $\begin{array}{l}\text { ApoC3 haplotype: }-482 \mathrm{C} /-455 \mathrm{~T} / \text { Intron I } \\
\text { G/Gly34Gly C/Sstl (3238) C }\end{array}$ & $45.5 \% 49$ & $10.8 \% 49$ \\
\hline Protease Inhibitor & $\begin{array}{l}\text { ApoC3 haplotype: }-482 \mathrm{C} /-455 \mathrm{C} / \text { Intron I } \\
\text { G/Gly34Gly C/Sstl (3238) C }\end{array}$ & $12.1 \% 49$ & $1.5 \%{ }^{49}$ \\
\hline Protease Inhibitor & $\begin{array}{l}\text { ApoC3 haplotype: }-482 \mathrm{C} /-455 \mathrm{~T} / \text { Intron I } \\
\text { G/Gly34Gly T/Sstl (3238) C }\end{array}$ & $10.5 \% 49$ & $6.3 \%{ }^{49}$ \\
\hline Protease Inhibitor & $\begin{array}{l}\text { ApoC3 haplotype: }-482 \mathrm{~T} /-455 \mathrm{C} / \text { Intron I } \\
\text { C/Gly34Gly C/Sstl (3238) C }\end{array}$ & $12.4 \% 49$ & $57.1 \% 49$ \\
\hline Protease Inhibitor & $\begin{array}{l}\text { ApoC3 haplotype: }-482 \mathrm{~T} /-455 \mathrm{C} / \text { Intron I } \\
\text { C/Gly34Gly T/Sstl (3238) G }\end{array}$ & $8.2 \%{ }^{49}$ & $8.2 \%{ }^{49}$ \\
\hline
\end{tabular}




\section{Discussion}

This review has discussed race-specific genetic variation at a number of loci which affects the tolerance and rate of adverse side-effects among HIV-infected patients undergoing standard HAART therapy. Individuals of African descent fare better than their Caucasian counterparts in terms of abacavir hypersensitivity reactions due to the fact that the prevalence of the HLA-B*5701 polymorphism is lower in this population. Clinical screening for HLA-B*5701 is currently utilized with much success. A number of studies however, have raised the question as to whether this type of screen is necessary and effective for individuals of African descent. A recent study by Saag and colleagues has suggested that this type of screening is in fact valuable and warranted for this population. ${ }^{12}$

Discontinuation of EFV- or NVP-based treatments due to adverse side-effects has been a major problem in individuals of African descent. This phenomenon has resulted in poor clinical outcomes for this population. Indeed, there is pharmacogenomic data suggesting that the genetics of this population lends them to experience elevated and prolonged exposure to EFV and NVP during treatment. This increased exposure leads to the increase in observed adverse events in these individuals. Further research should be conducted to find ways to make EFV and NVP treatment safer for this group.

Individuals of African descent enjoy more favorable lipid profiles than their white peers. However, it is worrisome and intriguing that this group appears to have the most marked increase in triglyceride levels during protease inhibitor treatment. Further research should be done to understand the molecular and genetic root for this phenomenon, as it may help to make treatment more efficacious and safe for all populations.

\section{Disclosures}

The authors report no conflicts of interest in this work.

\section{References}

1. Pirmohamed M, Back DJ. The pharmacogenomics of HIV therapy. Pharmacogenomics J. 2001;1(4):243-253.

2. Kayser S. Pharmacogenomics and the potential for personalized therapeutics in cardiovascular disease. Prog Cardiovasc Nurs. 2007;22(2):104-107.

3. Haas DW, Ribaudo HJ, Kim RB, et al. Pharmacogenetics of efavirenz and central nervous system side effects: an Adult AIDS Clinical Trials Group study. AIDS. 2004;18(18):2391-2400.

4. Hammer SM, Eron JJ Jr, Reiss P, et al; International AIDS Society-USA. Antiretroviral treatment of adult HIV infection: 2008 recommendations of the International AIDS Society-USA panel. JAMA. 2008;300(5): 555-570.

5. Palella FJ Jr. Delaney KM, Moorman AC, et al. Declining morbidity and mortality among patients with advanced human immunodeficiency virus infection. HIV Outpatient Study Investigators. $N$ Engl J Med. 1998;338(13):853-860.
6. Kilmarx PH. Global epidemiology of HIV. Curr Opin HIV AIDS. 2009;4(4):240-246.

7. Garber M. HIV-infected African Americans are willing to participate in HIV treatment trials. J Gen Intern Med. 2007;22(1):17-42.

8. Burroughs VJ, Maxey RW, Levy RA. Racial and ethnic differences in response to medicines: towards individualized pharmaceutical treatment. J Natl Med Assoc. 2002;94(10 Suppl):1-26.

9. Rodríguez-Nóvoa S, Barreiro P, Jiménez-Nacher I, Rendón A, Soriano V. Pharmacogenetics in HIV therapy. AIDS Rev. 2005;7(2): 103-112.

10. Pourpak Z, Fazlollahi MR, Fattahi F. Understanding adverse drug reactions and drug allergies: principles, diagnosis and treatment aspects. Recent Pat Inflamm Allergy Drug Discov. 2008;2(1):24-46.

11. Trowsdale J, Campbell RD. Complexity in the major histocompatibility complex. Eur J Immunogenet. 1992;19(1-2):45-55.

12. Saag M, Balu R, Phillips E, et al; Study of Hypersensitivity to Abacavir and Pharmacogenetic Evaluation Study Team. High sensitivity of human leukocyte antigen-b*5701 as a marker for immunologically confirmed abacavir hypersensitivity in white and black patients. Clin Infect Dis. 2008;46:1111-1118.

13. Mallal S, Phillips E, Carosi G, et al. HLA-B*5701 screening for hypersensitivity to abacavir. $N$ Engl J Med. 2008;358:568-579.

14. Hetherington S, Hughes AR, Mosteller M, et al. Genetic variations in HLA-B region and hypersensitivity reactions to abacavir. Lancet. 2002;359:1121-1122.

15. Hughes AR, Mosteller M, Bansal AT, et al; CNA30027 Study Team; CNA30032 Study Team. Association of genetic variations in HLA-B region with hypersensitivity to abacavir in some, but not all, populations. Pharmacogenomics. 2004;5(2):203-211.

16. Cressey TR, Lallemant M. Pharmacogenetics of antiretroviral drugs for the treatment of HIV-infected patients: an update. Infect Genet Evol. 2007;7(2):333-342.

17. Rauch A, Nolan D, Martin A, et al. Prospecitve genetic screening decreases the incidence of abacavir hypersensitivity reactions in the Western Australian HIV cohort study. Clin Infect Dis. 2006;43(1):99-102.

18. Mallal S, Nolan D, Witt C, et al. Association between presence of HLA-B*5701, HLA-DR7, and HLA-DQ3 and hypersensitivity to HIV-1 reverse-transcriptase inhibitor abacavir. Lancet. 2002;359(9308): 727-732.

19. Lallemant MJ, Le Coeur G, Mary S, et al. Single-dose perinatal nevirapine plus standard zidovudine to prevent mother-to-child transmission of HIV-1 in Thailand. N Engl J Med. 2004;351(3):217-228.

20. Zhou SF. Structure, function and regulation of P-glycoprotein and its clinical relevance in drug disposition. Xenobiotica. 2008;38(7-8): 802-832.

21. Cupp MJ, Tracy TS. Cytochrome P450: new nomenclature and clinical implications. Am Fam Physician. 1998;57(1):107-116.

22. Ende M, Sahtoe C, Dieleman J, Burger D, Schaik R. Prevalence of single nucleotide polymorphisms in MDR-1 and CYP3 A genes within a heterogenous HIV-1-infected population and effect on nevirapine plasma levels. San Francisco, CA: Conference on Retroviruses and Opportunistic Infections; 2004.

23. Haas DW, Bartlett JA, Andersen JW, et al; Adult AIDS Clinical Trials Group. Pharmacogenetics of nevirapine-associated hepatotoxicity: an Adult AIDS Clinical Trials Group collaboration. Clin Infect Dis. 2006;43(6):783-786.

24. Ritchie MD, Haas DW, Motsinger AA, et al. Drug transporter and metabolizing enzyme gene variants and nonnucleoside reverse transcriptase inhibitor hepatotoxicity. Clin Inf Dis. 2006;43: 779-782.

25. Haas DW, Smeaton LM, Shafer RW, et al. Pharmacogenetics of long-term responses to antiretroviral regimens containing Efavirenz and/or Nelfinavir: an Adult Aids Clinical Trials Group Study. J Infect Dis. 2005;192:1931-1942.

26. Saitoh A, Fletcher CV, Brundage R, et al. Efavirenz pharmacokinetics in HIV-1-infected children are associated with CYP2B6-G516T polymorphism. J Acquir Immune Defic Syndr. 2007;45(3):280-285. 
27. Ribaudo HJ, Haas DW, Tierney C, et al; Adult AIDS Clinical Trials Group Study. Pharmacogenetics of plasma efavirenz exposure after treatment discontinuation: an Adult AIDS Clinical Trials Group Study. Clin Infect Dis. 2006;42(3):401-407.

28. Friis-Møller N, Weber R, Reiss P, et al; DAD study group. Cardiovascular disease risk factors in HIV patients - association with antiretroviral therapy. Results from the DAD study. AIDS. 2003;17:1179-1193.

29. d'Arminio A, Sabin CA, Phillips AN, et al. Cardio- and cerebrovascular events in HIV-infected persons. AIDS. 2004;18:1811-1817.

30. Mary-Krause M, Cotte L, Simon A, Partisani M, Costagliola D. Increased risk of myocardial infarction with duration of protease inhibitor therapy in HIV-infected men. AIDS. 2003;17:2479-2486.

31. Fontas E, van Leth F, Sabin CA, et al. Lipid profiles in HIV-infected patients receiving combination antiretroviral therapy: Are different antiretroviral drugs associated with different lipid profiles? J Infect Dis. 2004;189:1056-1074.

32. Hui DY. Effects of HIV protease inhibitor therapy on lipid metabolism. Prog Lipid Res. 2003;42:81-92.

33. Grinspoon S, Carr A. Cardiovascular risk and body-fat abnormalities in HIV-infected adults. $N$ Engl J Med. 2005;352:48-62.

34. Bonnet E, Ruidavets JB, Tuech J, et al. Apoprotein c-III and E-containing lipoparticles are markedly increased in HIV-infected patients treated with protease inhibitors: Association with the development of lipodystrophy. J Clin Endocrinol Metab. 2001;86:296-302.

35. Cahn PE, Gatell JM, Squires K, et al. Atazanavir - a once-daily HIV protease inhibitor that does not cause dyslipidemia in newly treated patients: Results from two randomized clinical trials. J Int Assoc Physicians AIDS Care (Chic Ill). 2004;3:92-98.

36. Liang JS, Distler O, Cooper DA, et al. HIV protease inhibitors protect apolipoprotein B from degradation by the proteasome: A potential mechanism for protease inhibitor-induced hyperlipidemia. Nat Med. 2001;7:1327-1331.

37. Puro V. Effect of short-course of antiretroviral agents on serum triglycerides of healthy individuals. AIDS. 2000;14:2407-2408.

38. Olson RE. Discovery of the lipoproteins, their role in fat transport and their significance as risk factors. $J$ Nutr. 1998;128(2 Suppl): 439S-443S.
39. Segrest JP, Jones MK, De Loof H, Dashti N. Structure of Apolipoprotein B-100 in low denstiy lipoproteins. J Lipid Res. 2001;42(9): 1346-1367.

40. Surguchov AP, Page GP, Smith L, Patsch W, Boerwinkle E. Polymorphic markers in apolipoprotein C-III gene flanking regions and hypertriglyceridemia. Arterioscler Thromb Vasc Biol. 1996;16:941-947.

41. Li WW, Dammerman MM, Smith JD, Metzger S, Breslow JL, Leff T. Common genetic variation in the promoter of the human apo CIII gene abolishes regulation by insulin and may contribute to hypertriglyceridemia. J Clin Invest. 1995;96:2601-2605.

42. Dammerman M, Sandkuijl LA, Halaas JL, Chung W, Breslow JL. An apolipoprotein CIII haplotype protective against hypertriglyceridemia is specified by promoter and 3' untranslated region polymorphisms. Proc Natl Acad Sci U S A. 1993;90:4562-4566.

43. van Dijk KW, Rensen PC, Voshol PJ, Havekes LM. The role and mode of action of apolipoproteins CIII and AV: synergistic actors in triglyceride metabolism? Curr Opin Lipidol. 2004;15:239-246.

44. Dallinga-Thie GM, van Linde-Sibenius Trip M, Rotter JI, et al. Complex genetic contribution of the Apo AI-CIII-AIV gene cluster to familial combined hyperlipidemia. Identification of different susceptibility haplotypes. J Clin Invest. 1997;99:953-961.

45. Mahley RW, Rall SC Jr. Apolipoprotein E: far more than a lipid transport protein. Annu Rev Genomics Hum Genet. 2000;1:507-537.

46. Tarr PE, Taffe P, Bleiber G, et al. Modeling the influence of APOC3, APOE, and TNF polymorphisms on the risk of antiretroviral therapy associated lipid disorders. J Infect Dis. 2005;191:1419-1426.

47. Fauvel JB, Ruidavets E, Ferrieres JB, et al. An interaction between apo C-III variants and protease inhibitors contributes to high triglyceride/low HDL levels in treated HIV patients. AIDS. 2001;15(18): 2397-2406.

48. Gardner CD, Winkleby MA, Fortmann SP. Population frequency distribution of non-high-density lipoprotein cholesterol (Third National Health and Nutrition Examination Survey [NHANES III], 1988-1994). Am J Cardiol. 2000;86:299-304.

49. Foulkes AS, Wohl DA, Frank I, et al. Associations among race/ethnicity, ApoC-III genotypes, and lipids in HIV-1-infected individuals on antiretroviral therapy. PLoS Med. 2006;3(2):e52.

\section{Publish your work in this journal}

Pharmacogenomics and Personalized Medicine is an international, peer-reviewed, open access journal characterizing the influence of genotype on pharmacology leading to the development of personalized treatment programs and individualized drug selection for improved safety, efficacy and sustainability. This journal is indexed on the

\section{Dovepress}

American Chemical Society's Chemical Abstracts Service (CAS). The manuscript management system is completely online and includes a very quick and fair peer-review system, which is all easy to use. Visit http://www.dovepress.com/testimonials.php to read real quotes from published authors. 